\title{
Wideband Tri-band Filter Design using Stepped-impedance Stub Resonators
}

\author{
Mengmeng Cui ${ }^{1,2}$, Yun Liu ${ }^{3}$, Shengjun Xue ${ }^{1}$ and Jin Wang ${ }^{1}$ \\ ${ }^{1}$ Jiangsu Engineering Center of Network Monitoring, Nanjing University of \\ Information Science \& Technology, Nanjing, China \\ ${ }^{2}$ School of Applied Meteorological Science, Nanjing University of Information \\ Science \& Technology, Nanjing, China \\ ${ }^{3}$ College of Electronic and Information Engineering, Nanjing University of \\ Aeronautics and Astronautics, Nanjing, China \\ \{cuimengmeng,wangjin\}@nuist.edu.cn; yunliu@nuaa.edu.cn; sjxue@163.com
}

\begin{abstract}
This letter presents a wideband tri-passband filter in which tri-section stepped-impedance shunt-stubs are used as tri-mode resonators, connected by transmission lines. Developed from the topology of quarter-wavelength stub filters, these filters have much wider passbands compared to many other designs published. The stepped-impedance stubs also contribute transmission zeros between the passbands, thus increasing the isolation. A prototype filter with passbands located at 0.6-1, 1.5-1.8 and 2.2-2.4 GHz is designed by an optimization method. Good agreement between simulation and measurements in respect of low insertion loss and high isolation is demonstrated.
\end{abstract}

Keywords: Tri-band filters, stepped impedance stubs, J inverters

\section{Introduction}

Filters refer to the electrical components to reduce or eliminate the influence of harmonic on power system. It's a kind of device to eliminate the interference noise. The input or output after filtration is DC pure. Outside circuit, frequency of specific frequencies or the frequency of the frequency effectively filter is the filter, its function is to get a specific frequency or eliminating a specific frequency.

The main parameters of filters are the Center Frequency, the Cutoff Frequency, the Bandwidth, the Insertion Loss, the Ripple, the Passband Riplpe, the Return Loss and the Delay. Passbands filters allows a certain frequency signal. Signals, interferences and noises below or above the passbands are forbidden.

An ideal filter should have a completely flat passbands, no gain or attenuation in the passbands, and the band of all frequencies are completely decays away, in addition, through the transition zone and done in the frequency range minimum. In fact, there is no ideal bandpass filters. Filters can not be all frequency range completely attenuate expectations, especially in the pass band and a decay but not isolated range. This is usually referred to as 
the filter roll-off phenomenon, and the use of each of the ten frequency attenuation range of $\mathrm{dB}$ to represent. Usually, the filter design to ensure the roll-off range is narrow and better performance of this filters, closer and design.

Recently with the challenging demand of personal communications, multi-band systems that can support two or more systems with good isolation between bands are becoming important. Tri-band filters are one kind of key components in these systems. Various configurations have been proposed to realize tri-band filters. Thus [1] arranged three sets of resonators for realizing three passbands. For higher circuit efficiency, multi-mode resonators are adopted in tri-band filter design [2-4]. In [2], dual-mode and single mode resonators are combined to construct a tri-band filter. In [3] three-section stepped impedance resonator (SIR) technique are introduced for tri-band response with compact dimensions. A kind of stubloaded tri-mode resonator is proposed to construct tri-band filters with transmission zeroes between the passbands [4]. In most of the above, Input/output and internal couplings are parallel couplings which result in narrow passbands. In this letter, passbands are efficiently broadened by adopting a stub bandpass filter topology [5] with all inhomogeneous stubs replaced by tri-section stepped-impedance stubs.

Tri-section stepped-impedance stub and tri-band filter: A classical wideband filter topology is quarter-wavelength stub filter, in which $\lambda / 4$ shunt stubs are used as resonators while $\lambda / 4$ connecting lines are used to implement $\mathbf{J}$ inverters for coupling [5]. To realize wideband triband filters, we use tri-section stepped-impedance stubs as tri-mode resonators.

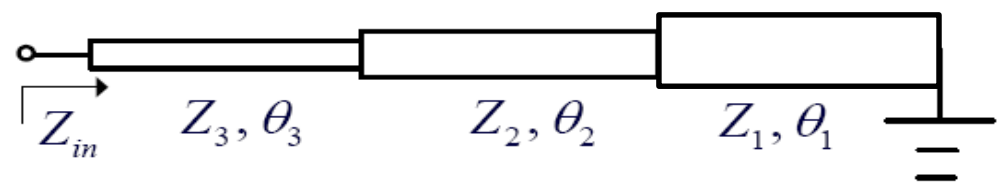

Figure 1. Tri-section stepped-impedance stub resonator

Figure 1 gives the circuit of a tri-section stepped impedance stub having the following input impedance:

$$
Z_{\text {in }}=j \frac{Z_{1} Z_{2} Z_{3} \tan \theta_{1}+Z_{2}^{2} Z_{3} \tan \theta_{2}+Z_{2} Z_{3}^{2} \tan \theta_{3}-Z_{1} Z_{3}^{2} \tan \theta_{1} \tan \theta_{2} \tan \theta_{3}}{Z_{2} Z_{3}-Z_{1} Z_{3} \tan \theta_{1} \tan \theta_{2}-Z_{1} Z_{2} \tan \theta_{1} \tan \theta_{3}-Z_{2}^{3} \tan \theta_{2} \tan \theta_{3}}
$$

Here $\theta_{1}, \theta_{2}$ and $\theta_{3}$ are frequency dependent electrical lengths. Resonances occur when $Z_{\text {in }}$ is infinite while transmission zeros appear at the frequencies where $Z_{\text {in }}$ is zero. 


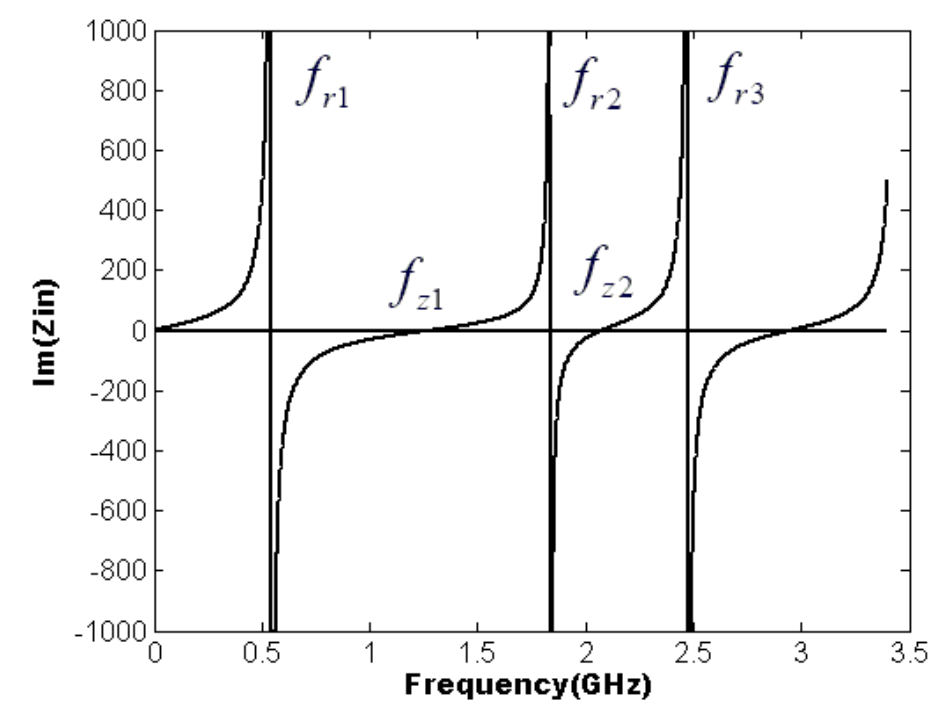

Figure 2. Plot of the input reactance of a tri-section stepped-impedance stub resonator

Figure 2 is the plot of $\operatorname{Zin}(\mathrm{f})$, in which we can see two transmission zeros are distributed between three resonating frequencies:

$$
f_{r 1}<f_{z 1}<f_{r 2}<f_{z 2}<
$$

Each stepped-impedance stub should be designed so that three resonating frequencies are located within three passbands respectively thus contributing one transmission pole for each passband. Additionally, correct admittance slopes at the resonating frequencies are required for realizing specified passbands.

An admittance or $\mathbf{J}$ inverter is an ideal inverter with an electric length of 90 degrees at all frequencies. Homogeneous transmission lines with fixed lengths can not act as ideal $\mathbf{J}$ inverters at all three passbands, so some passbands will have frequency shift and bandwidth variation compared to those desired. These can be compensated by adjusting the circuit parameters of all the tri-section stubs. To achieve good passbands, an optimizing method is utilized for extracting the circuit parameters.

\section{Tri-section Stepped-impedance Stub and Tri-band Filter}

A tri-band filter with passbands of 0.6-1.0, 1.5-1.8 and 2.2-2.4GHz (fractional bandwidths of $50 \%, 18 \%, 9 \%$ ) is designed and fabricated. Figure 3 shows the configuration of the triband filter, whose parameters are extracted using the optimizing tools integrated in Ansoft Serenade 8.7. 


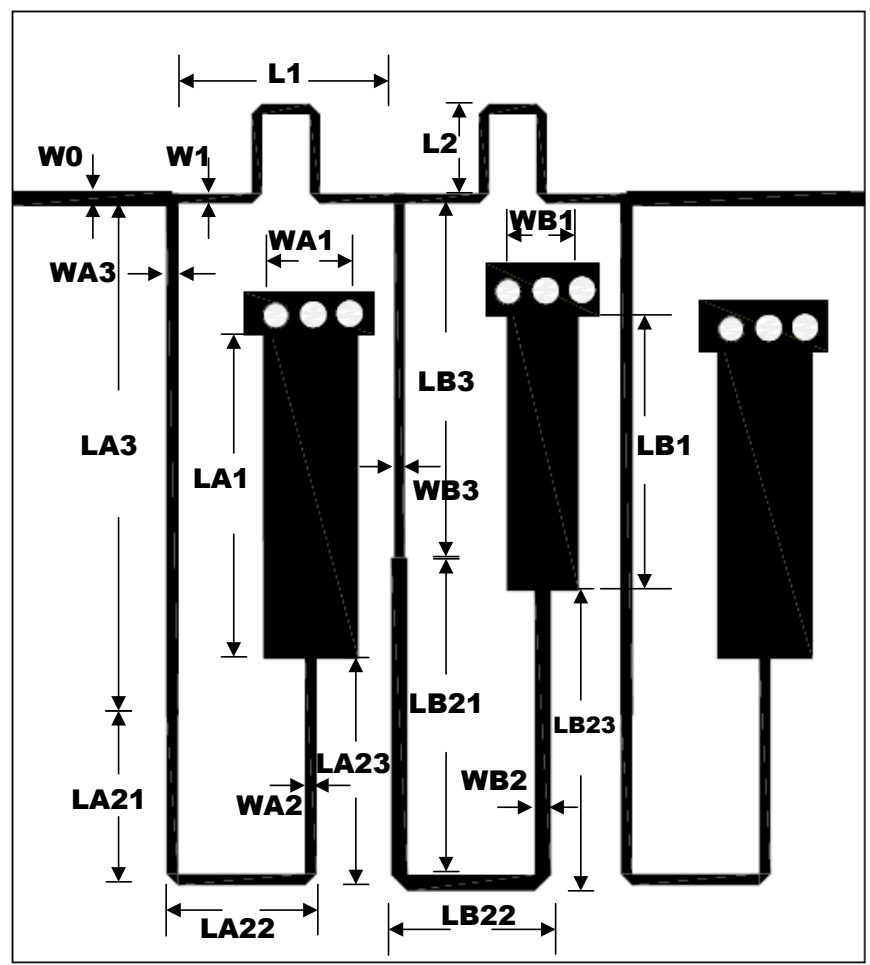

Figure 3. Topology and dimensions of the proposed tri-band filter

This is a three-order tri-band filter in which three tri-section stepped-impedance stubs are connected by two transmission lines with symmetrical dimensions. To realize compact size, folded and meandered transmission lines are used. This filter is fabricated on a substrate with a thickness of $0.406 \mathrm{~mm}$ and a dielectric constant $\varepsilon_{r}$ of 3.38. All the dimensions designed are given as follows(unit: $\mathrm{mm}$ ): $\quad W_{0}=0.92, \quad W=0.64, \quad W A 1=6, \quad W 2=0.71, \quad W A B=0.78$, $V B 1=4.55, \quad V B 2=1.02, \quad L B 3=0.65, \quad L 1=13.95, \quad L 2=5.64, \quad L A 1=20.44, \quad L A 21=10.71$, $\angle A 22=9.42, \angle A 23=14.26, \quad L B 1=17.33, \angle B 21=20, \angle B 22=10.04, \quad \angle B 23=18.92, \quad L B 3=22.3 . \quad$ Each stepped-impedance stub is grounded at the end with three via holes whose diameters are $0.8 \mathrm{~mm}$. The total size of the filter is $53.6 \times 60 \mathrm{~mm} 2$.

The circuit simulation results and the measured responses are compared in Figure 4, and good agreement is observed. The lowest insertion losses of the three passbands are $0.3 \mathrm{~dB}$ 、 $0.6 \mathrm{~dB}$ and $0.9 \mathrm{~dB}$ respectively, and each passband has return loss over $15 \mathrm{~dB}$. Transmission zeros are allocated between the passbands, and thus high isolations of $28 \mathrm{~dB}$ and $40 \mathrm{~dB}$ are achieved. There is frequency shift between the simulated and measured plots due to fabrication tolerances. 


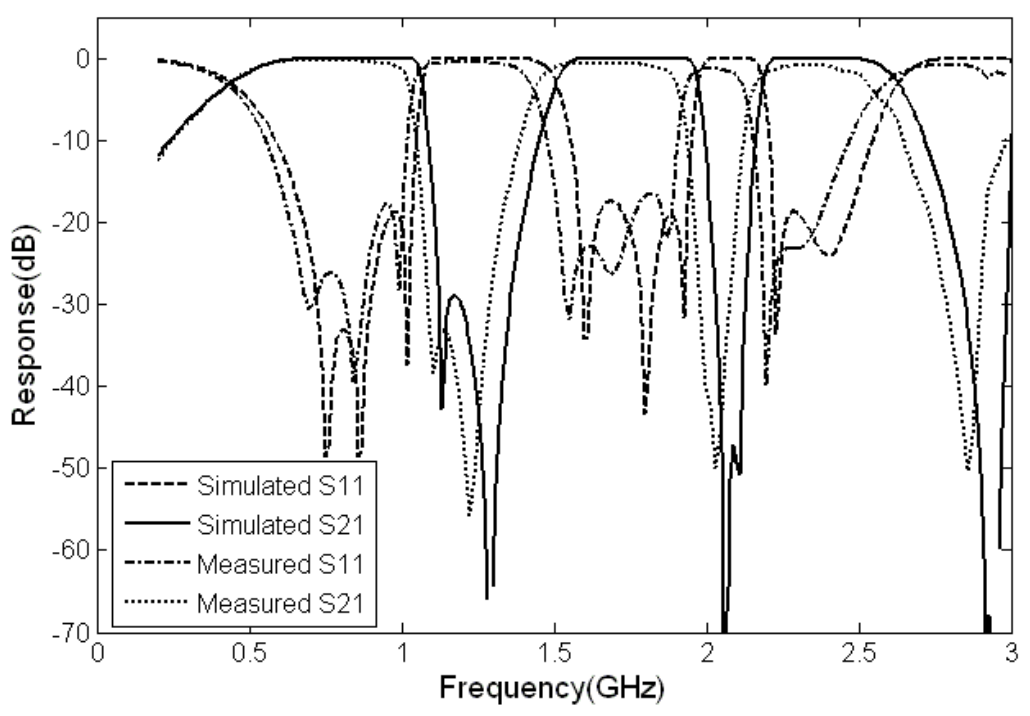

Figure 4. Compared simulation and measurement results of the tri-band filter

\section{Results and Discussion}

We presents a wideband tri-passband filter in which tri-section stepped-impedance shuntstubs are used as tri-mode resonators, connected by transmission lines. Developed from the topology of quarter-wavelength stub filters, these filters have much wider passbands compared to many other designs published. The stepped-impedance stubs also contribute transmission zeros between the passbands, thus increasing the isolation. A prototype filter with passbands located at $0.6-1,1.5-1.8$ and $2.2-2.4 \mathrm{GHz}$ is designed by an optimization method.

The wideband tri-band filter realization is proposed by adopting stub filter topology and tri-section stepped-impedance stub resonators. A prototype filter was designed using an optimization technique, fabricated and measured. Experiment results demonstrate good performance features, including wide passbands (50\%, 18\% and 9\%), low insertion loss, high inter-band isolation and compact dimensions.

\section{References}

[1] C. F. Chen, T. Y. Huang and R. B. Wu, "Design of dual- and triplepassband filters using alternately cascaded multiband resonators”, IEEE Trans. Microw. Theory Tech., vol. 54, no. 9, (2006) September, pp. 3550-3558.

[2] C. -I. G. Hsu, C. -H. Lee and Y. -H. Hsieh, "Tri-band bandpass filter With sharp passband skirts designed using tri-section SIRs", IEEE Microw. Wireless Compon. Lett., vol. 18, no. 1, (2008) January, pp. 19-21.

[3] F. C. Chen and Q. X. Chu, "Design of compact tri-band bandpass filters using assembled resonators", IEEE Trans. Microw. Theory Tech., vol. 57, no. 1, (2009) January, pp. 165-171.

[4] F. C. Chen and Q. X. Chu, "Tri-band bandpass filters using stub loaded resonators", Electron. Lett., vol. 44, no. 12, (2008) June, pp. 747-749.

[5] J. -S. Hong and M. J. Lancaster, "Microstrip Filter for RF/Microwave Applications", Wiley, New York, (2001).

[6] J. García-García, J. Bonache and F. Martín, "Application of e-lectromagnetic bandgaps to the design of ultrawide band-pass filters with good out-of-band performance”, IEEETrans Microw Theory Tech., (2006).

[7] H. Shaman and J. Hong, "Input and output cross-coupled wide-band bandpass filter", IEEE Trans Microw Theory Tech., (2007).

[8] F. Wei, Q. Wu, X. Shi and L. Chen, "Compact UWB bandpassfilter with dual notched bands based on SCRLH resonator", IEEE Microw Wirel Compon Lett., (2011). 
[9] P. Cai, Z. Ma, X. Guan, X. Yang, Y. Kobayashi, T. Anada and G. Hagiwara, "A compact UWB bandpass filter using two section open-circuited stubs to realize transmission zeros", Proc. Asia-Pasific Microw. Conf. (APMC), (2005).

[10] L. Zhu, S. Sun and W. Menzel, "Ultra-wideband (UWB) bandpass filters using multiple-mode resonator", IEEE Microw. Wireless Compon. Lett., (2005).

[11] Z. C. Hao and J. S. Hong, "Ultra-wideband bandpass filter using multilayer liquid-crystal-polymer technology", IEEE Trans. Microw. Theory Tech., (2008).

[12] J. -S. Hong and M. J. Lancaster, "Microstrip filters for RF/microwave applications, (2001).

\section{Authors}

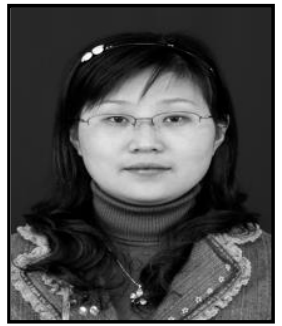

Mengmeng Cui obtained the B.S. degree in the Nanjing Normal University, China in 2002. She received her M.S. degree in Nanjing University of Information Science \& Technology, China in 2007. She is pursuing here Ph.D. degree. Now, she is a lecturer in Nanjing University of Information Science \& technology, China. Her main research interests include optimization algorithms and optimizational synthesis of microwave filters.

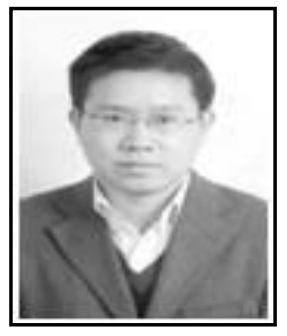

Yun Liu received the B.S and M.S degrees in radio physics from Nanjing University, Nanjing, China, respectively in 2000 and 2004, and got his PhD degree from the state key laboratory of millimeter waves, Southeast University, Nanjing, China in 2009. He joined Nanjing University of Aeronautics and Astronautics in 2009, and is currently an associate professor. His main research interest includes microwave filters, passive components.

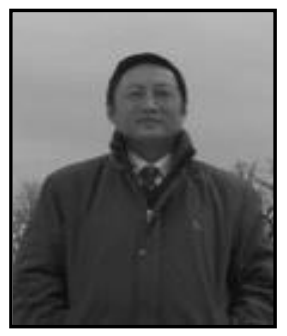

Shengjun Xue obtained the B.S. degree in the Zhejiang University, China in 1983. He received his Ph.D. degree in Wuhan University of Technology in 2000. Now, she is a professor in Nanjing University of Information Science \& technology, China. 